\title{
Correction: Liu, Y.-S.; et al. Inhibition of Protein Phosphatase 1 Stimulates Noncanonical ER Stress eIF2 $\alpha$ Activation to Enhance Fisetin-Induced Chemosensitivity in HDAC Inhibitor-Resistant Hepatocellular Carcinoma Cells. Cancers 2019, 11, 918
}

\author{
Yi-Sheng Liu ${ }^{1,2}$, Yu-Chun Chang ${ }^{3,4}$, Wei-Wen Kuo ${ }^{5} \mathbb{0}$, Ming-Cheng Chen ${ }^{6,7}{ }^{\text {, Hsi-Hsien Hsu }}{ }^{8,9}$, \\ Chuan-Chou Tu ${ }^{10}$, Yu-Lan Yeh 11,12, Vijaya Padma Viswanadha ${ }^{13}$, Po-Hsiang Liao 14,15,16,*(D) and \\ Chih-Yang Huang 15,16,17,18,*iD \\ 1 Program for Aging, China Medical University, Taichung 404, Taiwan; menace0712@yahoo.com.tw \\ 2 Division of Hematology and Oncology, Department of Medicine, Kaohsiung Armed Forces General Hospital, \\ Kaohsiung 802, Taiwan \\ 3 Graduate Department of Biological Science and Technology, National Pingtung University of Science and \\ Technology, Pingtung 912, Taiwan; dye60136@gmail.com \\ 4 School of Chinese Medicine, China Medical University, Taichung 404, Taiwan \\ 5 Department of Biological Science and Technology, China Medical University, Taichung 404, Taiwan; \\ wwkuo@mail.cmu.edu.tw \\ 6 Division of Colorectal Surgery, Department of Surgery, Taichung Veterans General Hospital, \\ Taichung 407, Taiwan; claudiochen7@gmail.com \\ 7 Faculty of Medicine, National Yang-Ming University, Taipei 112, Taiwan \\ 8 Division of Colorectal Surgery, MacKay Memorial Hospital, Taipei 104, Taiwan; hsu5936@ms3.hinet.net \\ MacKay Medicine, Nursing and Management College, Taipei 112, Taiwan \\ 10 Division of Chest Medicine, Department of Internal Medicine, Armed Force Taichung General Hospital, \\ Taichung 411, Taiwan; tu4697@gmail.com \\ 11 Department of Pathology, Changhua Christian Hospital, Changhua 500, Taiwan; 1867@cch.org.tw \\ 12 Department of Medical Technology, Jen-Teh Junior College of Medicine, Nursing and Management, \\ Miaoli 356, Taiwan \\ 13 Department of Biotechnology, Bharathiar University, Coimbatore 641046, India; \\ vvijayapadma@rediffmail.com \\ 14 Graduate Institute of Basic Medical Science, China Medical University, Taichung 404, Taiwan \\ 15 Cardiovascular Research Center, Hualien Tzu Chi Hospital, Hualien 970, Taiwan \\ 16 Center of General Education, Buddhist Tzu Chi Medical Foundation, Tzu Chi University of Science and \\ Technology, Hualien 970, Taiwan \\ 17 Department of Medical Research, China Medical University Hospital, China Medical University, \\ Taichung 404, Taiwan \\ 18 Department of Biotechnology, Asia University, Taichung 413, Taiwan \\ * Correspondence: robert750927@hotmail.com (P.-H.L.); cyhuang@mail.cmu.edu.tw (C.-Y.H.); \\ Tel.: +886-4-22052121 (ext. 7701) (P.-H.L.); +886-4-22053366 (ext. 3313) (C.-Y.H.)
}

Received: 22 September 2020; Accepted: 26 October 2020; Published: 3 November 2020

It has been reported that two sets of blot figures in Figure $3 \mathrm{~A}, \mathrm{C}$ looked identical in the original published paper [1]. After a careful check, the authors found that the p-eIF2 $\alpha$ band in Figure 3C was mistakenly uploaded, while the other bands are correct. The authors state this was due to carelessness.

With the approval of the Editor-in-Chief and the Editorial Office, the authors wish to replace Figure $3 C$ in [1] with an updated version.

The original version of Figure $3 \mathrm{C}$ is as follows: 


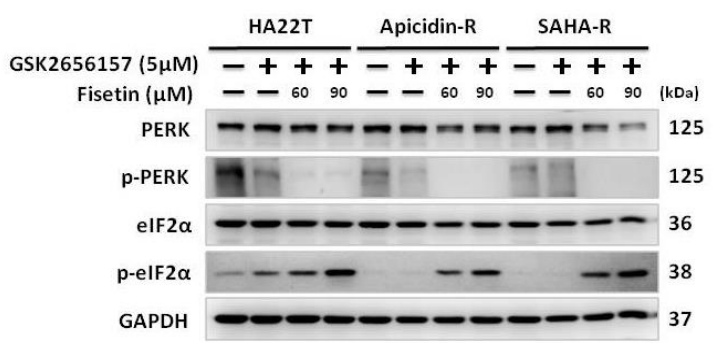

and should be replaced with the following version:

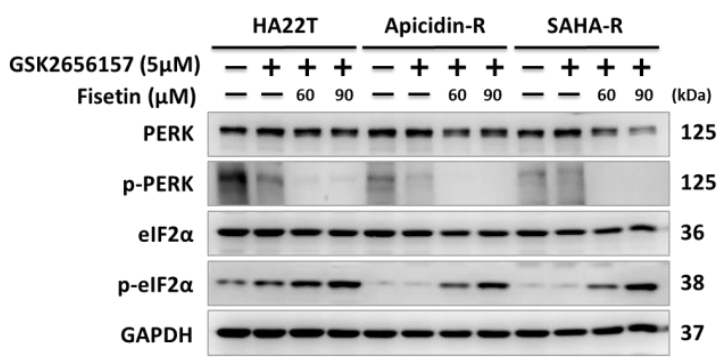

The authors apologize for any inconvenience caused and state that the scientific conclusions are unaffected. The original article has been updated.

\section{Reference}

1. Liu, Y.-S.; Chang, Y.-C.; Kuo, W.W.; Chen, M.C.; Hsu, H.H.; Tu, C.C.; Yeh, Y.L.; Viswanadha, V.P.; Liao, P.H.; Huang, C.-Y. Inhibition of protein phosphatase 1 stimulates noncanonical er stress eif2alpha activation to enhance fisetin-induced chemosensitivity in hdac inhibitor-resistant hepatocellular carcinoma cells. Cancers 2019, 11, 918. [CrossRef]

Publisher's Note: MDPI stays neutral with regard to jurisdictional claims in published maps and institutional affiliations.

(C) 2020 by the authors. Licensee MDPI, Basel, Switzerland. This article is an open access article distributed under the terms and conditions of the Creative Commons Attribution (CC BY) license (http://creativecommons.org/licenses/by/4.0/). 